\title{
Magnetic Properties of Mixed Spin Ising Diamond Chain
}

\section{Ma Xiao-Yu}

School of Science, Shenyang University of Technology, Shenyang, China

\section{Email address:}

1208924339@qq.com

\section{To cite this article:}

Ma Xiao-Yu. Magnetic Properties of Mixed Spin Ising Diamond Chain. Earth Sciences. Vol. 4, No. 6, 2015, pp. 280-285. doi: 10.11648/j.earth.20150406.20

\begin{abstract}
In this paper, we establish a mixed spin Ising diamond chain model on the basis of the effective-field theory with correlations. The formula of the magnetization and the magnetic susceptibility has been given. Effects of the various parameters such as exchange couplings and the anisotropy on the magnetization and the magnetic susceptibility of Ising diamond chain have been studied using numerical calculation. The results show that magnetization curves appear magnetization plateaus. The numbers of magnetization plateaus change with the exchange couplings and the anisotropy. The magnetic susceptibility changes with the exchange couplings, the anisotropy and the external magnetic field.
\end{abstract}

Keywords: The Effective-Field Theory with Correlations, Diamond Chain, The Anisotropy, The Magnetization, The Magnetic Susceptibility

\section{混自旋Ising钻石链的磁化特性研究}

\author{
马晓宇 \\ 理学院, 沈阳工业大学, 沈阳市, 中国

\section{邮箱} \\ 1208924339@qq. com
}

\begin{abstract}
摘要：本文在该模型基础上利用相关有效场理论，在考虑晶场的作用下，建立混自旋Ising钻石链模型，推导出系统 的磁矩和磁化率的计算公式, 利用数值计算研究各种参数如交换作用和晶场等对Ising钻石链的磁矩和磁化率的影响。 结果表明, 磁矩曲线会出现磁化平台效应, 磁化曲线的平台随着交换作用、晶场的不同发生变化。磁矩、磁化率曲线 也随交换作用、晶场和外磁场发生不同变化。
\end{abstract}

关键词: 相关有效场理论, 钻石链, 晶场, 磁矩, 磁化率

\section{1. 引言}

近年来, 低维磁性材料的研究引起了科学家们的极大 兴趣。一些有机聚合物和碱土含铜氧化物如 $\mathrm{Cu}_{3}\left(\mathrm{CO}_{3}\right)_{2}(\mathrm{OH})_{2}, \mathrm{BaCu}_{3} \mathrm{O}_{4}, \mathrm{Cu}_{3}\left(\mathrm{TeO}_{3}\right)_{2} \mathrm{Br}$ 和 $\mathrm{Bi}_{4} \mathrm{Cu}_{3} \mathrm{~V}_{2} \mathrm{O}_{14}$ 显示磁有 序, 这极大的吸引了理论和实验研究学者的注意 $[1,2]$ 。 这些材料在磁学研究中都被抽象成一种一维钻石链模型, 其中混自旋 $2,1 / 2$ 的反铁磁Ising钻石链的磁化强度研究 的最为广泛。在低温情况下, Xin等人模拟出了在磁场作
用下的不同的反铁磁交换作用的磁化强度, 发现了 dimmer 态和trimmer态 [3]。Okamoto等人用简并微扰理论和密度 矩阵重整化方法研究了 $\mathrm{Bi}_{4} \mathrm{Cu}_{3} \mathrm{~V}_{2} \mathrm{O}_{14}$ 和蓝铜矿 $\mathrm{Cu}_{3}\left(\mathrm{CO}_{3}\right)_{2}(\mathrm{OH})_{2}$ 。 在温度为零时, 反铁磁不对称钻石链的磁化出现阶梯效应 [4]。Drillon等人计算了 $\mathrm{A}_{3} \mathrm{Cu}_{3}\left(\mathrm{PO}_{4}\right)_{4}(\mathrm{~A}=\mathrm{Ca}, \mathrm{Sr})$ 的磁化 率, 比热和磁化强度 [5]。Honecker研究了在无磁场情况 下的非弹性中子散射蓝铜矿一般钻石链模型的动力和热 力学性质, 提供了磁热效应的数值数据并预测在略高于 30T的磁场中绝热磁化下应该观察到蓝铜矿的强冷却现象 [6]。Jiang等人用精确对角化方法研究了不对称混自旋 
$(1,1 / 2)$ 钻石链反铁磁相互作用的磁化特性 [7]。Cheng等 人研究了在有限温度下考虑外磁场的作用, 自旋为 $-1 / 2$ 的亚铁磁钻石链的磁性和热力学性质 [8]。Lisnyi 等人精 确求解了混自旋- $(1,1 / 2)$ Ising 钻石链的基态相图和磁化 过程 [9], 证明了交换作用影响下的混自旋对称和不对称 钻石链都会发生两个不同的磁化过程, 低温的磁化曲线至 少有两个不同的磁化平台。Ananikian等人将天然材料蓝 铜矿近似的抽象成不对称钻石链模型, 研究其磁化特性和 量子纠缠 $[10]$ 。

尽管钻石链系统已经取得了重大成绩, 有机聚合物和 碱土含铜氧化物磁性产生的机理仍然有待进一步揭示。这 类材料的研究大都没有涉及各向异性, 与之相关的理论也 鲜有报道。因此, 基于曾经利用相关有效场理论研究各向
异性的亚铁磁纳米粒子的磁化特性并发现阶梯效应现象 [11], 在此重点研究各向异性对混自旋 $2,1 / 2$ 和 $3 / 2$ 的钻 石链的磁化强度的影响, 进而研究低温情况下磁矩和磁化 率曲线随各参数的变化规律。

\section{2. 模型和方法}

混自旋Ising钻石链的自旋模型如图1所示。图中 $\mathrm{J}_{1}$ 表 示子格 $\mathrm{A}$ 和子格B之间的交换作用, $\mathrm{J}_{2}$ 表示子格 $\mathrm{A}$ 和子格 $\mathrm{C}$ 之 间的交换作用， $\mathrm{J}_{3}$ 表示子格 $\mathrm{B}$ 和子格 $\mathrm{C}$ 之间的交换作用。考 虑到外加磁场和晶场的影响, 系统的哈密顿量如式 (1) 所 示:

$$
\begin{aligned}
H & =\sum_{i=1}^{N} J_{1}\left(S_{i}^{z} \sigma_{i 1}^{z}+S_{i}^{z} \sigma_{i+1,1}^{z}\right)+\sum_{i=1}^{N} J_{2}\left(S_{i}^{z} \sigma_{i 2}^{z}+S_{i}^{z} \sigma_{i+1,2}^{z}\right) \\
& +\sum_{i=1}^{N}\left[J_{3} \sigma_{i 1}^{z} \sigma_{i 2}^{z}+h\left(S_{i}^{z}+\sigma_{i 1}^{z}+\sigma_{i 2}^{z}\right)\right]+\sum_{i=1}^{N} D_{1}\left(S_{i}^{z}\right)^{2}+\sum_{i=1}^{N} D_{2}\left(\sigma_{i 2}^{z}\right)^{2}
\end{aligned}
$$

其中, $S_{\mathrm{i}}^{z}\left(S_{i}^{x}\right), \sigma_{i 1}^{z}\left(\sigma_{i 1}^{x}\right)$ 和 $\sigma_{\mathrm{i} 2}^{z}\left(\sigma_{i 2}^{x}\right)$ 分别 为系统中 $A 、 B$ 和 $C$ 子格的 $\mathrm{z}(\mathrm{x})$ 方向自旋, 其有效自旋分 别为 $2 、 1 / 2,3 / 2$ 。系统的子格子总数为 $3 \mathrm{~N}_{0}$ 。 $\mathrm{D}_{1}$ 和 $\mathrm{D}_{2}$ 分别是 子格 $\mathrm{A}$ 和 $\mathrm{C}$ 的晶场, $\mathrm{h}$ 是 $\mathrm{z}$ 方向的外磁场。

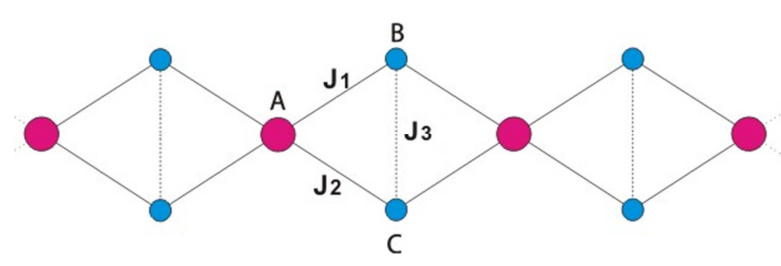

图1 混自旋Ising钻石链模型。

基于利用相关有效场理论，得到Ising钻石链模型的 三个子格 $\mathrm{A} 、 \mathrm{~B}$ 和 $\mathrm{C}$ 的磁矩Ma、 Mb 和Mc分别为:

$$
\begin{aligned}
& M_{a}=\left[\cosh \left(\frac{1}{2} J_{1} \nabla\right)+2 \times M_{b} \sinh \left(\frac{1}{2} J_{1} \nabla\right)\right]^{2} \\
& \times\left.\left[\cosh \left(\frac{1}{2} J_{2} \nabla\right)+2 \times M_{c} \sinh \left(\frac{1}{2} J_{2} \nabla\right)\right]^{2} F_{1}(x)\right|_{x=0} \\
& M_{b}=\left[\cosh \left(\eta J_{1} \nabla\right)+\frac{M_{a}}{\eta} \sinh \left(\eta J_{1} \nabla\right)\right]^{2} \\
& \times\left.\left[\cosh \left(\frac{1}{2} J_{3} \nabla\right)+2 \times M_{c} \sinh \left(\frac{1}{2} J_{3} \nabla\right)\right] F_{2}(x)\right|_{x=0} \\
& F_{1}(x)=\frac{4 \sinh [2 \beta(x+h)]+2 \exp \left(-3 D_{1} \beta\right) \sinh [\beta(x+h)]}{2 \cosh [2 \beta(x+h)]+2 \exp \left(-3 D_{1} \beta\right) \cosh [\beta(x+h)]+\exp \left(-4 D_{1} \beta\right)} \quad S_{\mathrm{i}}^{z}=2 \\
& F_{2}(x)=\frac{1}{2} \tanh \left[\frac{1}{2} \beta(x+h)\right] \quad \sigma_{i 1}^{z}=1 / 2
\end{aligned}
$$

$$
\begin{aligned}
M_{c} & =\left[\cosh \left(\eta J_{2} \nabla\right)+\frac{M_{a}}{\eta} \sinh \left(\eta J_{2} \nabla\right)\right]^{2} \\
& \times\left.\left[\cosh \left(\frac{1}{2} J_{3} \nabla\right)+2 \times M_{b} \sinh \left(\frac{1}{2} J_{3} \nabla\right)\right] F_{3}(x)\right|_{x=0}
\end{aligned}
$$

其中 $\nabla=\partial / \partial x$ 为系统的微分算符, 与之相对应的 $\eta_{1}{ }^{2}$ 和 $\eta_{2}^{2}$ 分别为:

$$
\begin{aligned}
\eta_{1}^{2}= & {\left[\cosh \left(\frac{1}{2} J_{1} \nabla\right)+2 \times M_{b} \sinh \left(\frac{1}{2} J_{1} \nabla\right)\right]^{2} } \\
& \times\left.\left[\cosh \left(\frac{1}{2} J_{2} \nabla\right)+2 \times M_{c} \sinh \left(\frac{1}{2} J_{2} \nabla\right)\right]^{2} G_{1}(x)\right|_{x=0} \\
\eta_{2}{ }^{2}= & {\left[\cosh \left(\eta J_{2} \nabla\right)+\frac{M_{a}}{\eta} \sinh \left(\eta J_{2} \nabla\right)\right]^{2} } \\
& \times\left.\left[\cosh \left(\frac{1}{2} J_{3} \nabla\right)+2 \times M_{b} \sinh \left(\frac{1}{2} J_{3} \nabla\right)\right] G_{3}(x)\right|_{x=0}
\end{aligned}
$$

函数 $F_{1}(x), F_{2}(x), F_{3}(x), G_{1}(x)$ 和 $G_{3}(\mathrm{x})$ 的表达 


$$
\begin{gathered}
F_{3}(x)=\frac{3 \sinh \left[\frac{3}{2} \beta(x+h)\right]+\exp \left(-2 D_{2} \beta\right) \sinh \left[\frac{1}{2} \beta(x+h)\right]}{2 \cosh \left[\frac{3}{2} \beta(x+h)\right]+2 \exp \left(-2 D_{2} \beta\right) \cosh \left[\frac{1}{2} \beta(x+h)\right]} \quad \sigma_{\mathrm{i} 2}^{z}=3 / 2 \\
G_{1}(x)=\frac{8 \cosh [2 \beta(x+h)]+2 \exp \left(-3 D_{1} \beta\right) \cosh [\beta(x+h)]}{2 \cosh [2 \beta(x+h)]+2 \exp \left(-3 D_{1} \beta\right) \cosh [\beta(x+h)]+\exp \left(-4 D_{1} \beta\right)} \quad S_{\mathrm{i}}^{z}=2 \\
G_{3}(x)=\frac{9 \cosh \left[\frac{3}{2} \beta(x+h)\right]+\exp \left(-2 D_{2} \beta\right) \cosh \left[\frac{1}{2} \beta(x+h)\right]}{4 \cosh \left[\frac{3}{2} \beta(x+h)\right]+4 \exp \left(-2 D_{2} \beta\right) \cosh \left[\frac{1}{2} \beta(x+h)\right]} \sigma_{\mathrm{i} 2}^{z}=3 / 2
\end{gathered}
$$

总的平均磁矩M计算公式如式 (12) :

$$
M=\left(M_{a}+2 M_{b}+2 M_{c}\right) / 5
$$

\section{3. 结果和讨论}

假设A、B和C子格的初始自旋值 $S_{i}^{z}=2, \sigma_{i 1}^{z}=1 / 2$ 和 $\sigma_{i 2}^{z}$ $=3 / 2$ 为正向, 自旋 $S_{i}^{z}$ 的方向沿外磁场正方向, 以交换作 用 $\mathrm{J}_{3}=1$ 为单位。

\section{1. 各物理参量对阶梯效应的影响}

图2 (a) - (c) 给出了交换作用 $\mathrm{J}_{1} / \mathrm{J}_{3}$ 取 $0.3 、 0.9$ 和 1.8 时 系统磁矩随外磁场的变化曲线, 其它参数选择为: $\mathrm{k}_{\mathrm{B}} \mathrm{T} / \mathrm{J}_{3}=0.05, \mathrm{~J}_{2} / \mathrm{J}_{3}=0.2, \mathrm{D}_{1} / \mathrm{J}_{3}=-2.1, \mathrm{D}_{2} / \mathrm{J}_{3}=-2.5$ 。
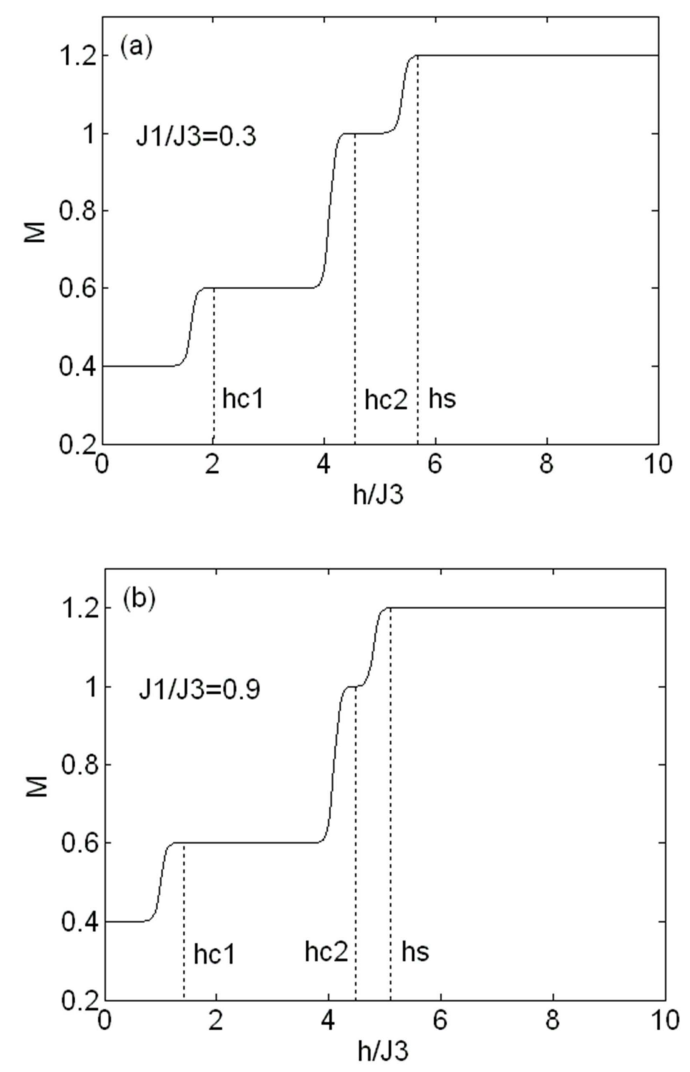

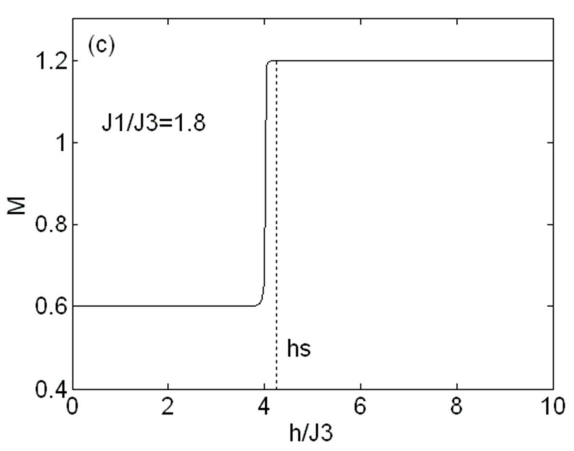

图2 改变 $\mathrm{J} 1 / \mathrm{J} 3$ 时的磁矩。

从图中可以看到, 每条磁化曲线都表现出阶梯效应 (磁化平台), 另外在磁化平台之间发生转变时, 虚线对 应的外磁场叫做临界磁场hc和饱和磁场hs. 图2 (a) 中可 以看到, 当 $\mathrm{J}_{1} / \mathrm{J}_{3}=0.3<\mathrm{J}_{3}=1$ 时有四个磁化平台, 即 $\mathrm{M}=0.4$, $0.6,1,1.2$ 。对应的临界磁场hc为 $2.03,4.58$ 和饱和磁 场hs 为5.73。图2 (b) 中可以看到, 当增加 $\mathrm{J}_{1} / \mathrm{J}_{3}$ 到 0.9 时, 磁化曲线仍为四个平台, 但是第一个临界磁场hc和饱和磁 场hs却都减小了, 分别为 $h c=1.43$ 和 $h s=5.13$, 而第二个临 界磁场hc保持不变。图2 (c) 中选择A和B之间交换作用 $\mathrm{J}_{1} / \mathrm{J}_{3}=1.8>\mathrm{J}_{3}=1$ 时两个磁化平台 $(\mathrm{M}=0.4,1)$ 消失, 只剩 两个磁化平台 $\mathrm{M}=0.6$ 和 1.2 , 此时只有一个饱和磁场 $h c=4.25$, 研究结果表明交换作用 $\mathrm{J}_{1} / \mathrm{J}_{3}$ 影响磁矩曲线平台 的个数, 也就是影响子晶格的自旋态。

图3 (a)-(d) 给出了磁晶各向异性 $\mathrm{D}_{1} / \mathrm{J}_{3}$ 取 $-0.5,-3.0$ 和 -4.0 时系统平均磁矩和三个子格磁矩随外磁场的变化 曲线, 其它参数选择为: $\mathrm{k}_{\mathrm{B}} \mathrm{T} / \mathrm{J}_{3}=0.05, \mathrm{~J}_{2} / \mathrm{J}_{3}=0.3, \mathrm{~J}_{2} / \mathrm{J}_{3}=0.2$, $\mathrm{D}_{2} / \mathrm{J}_{3}=-2.5$ 。

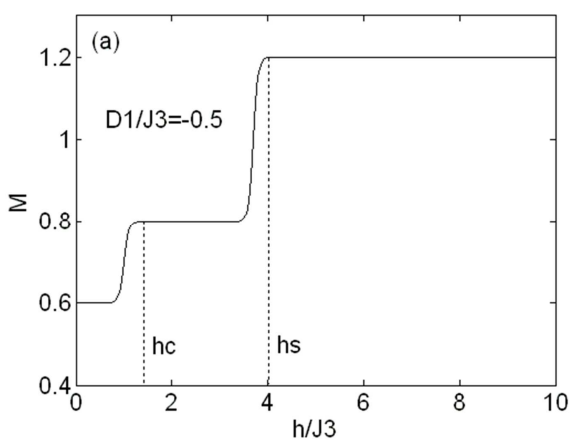



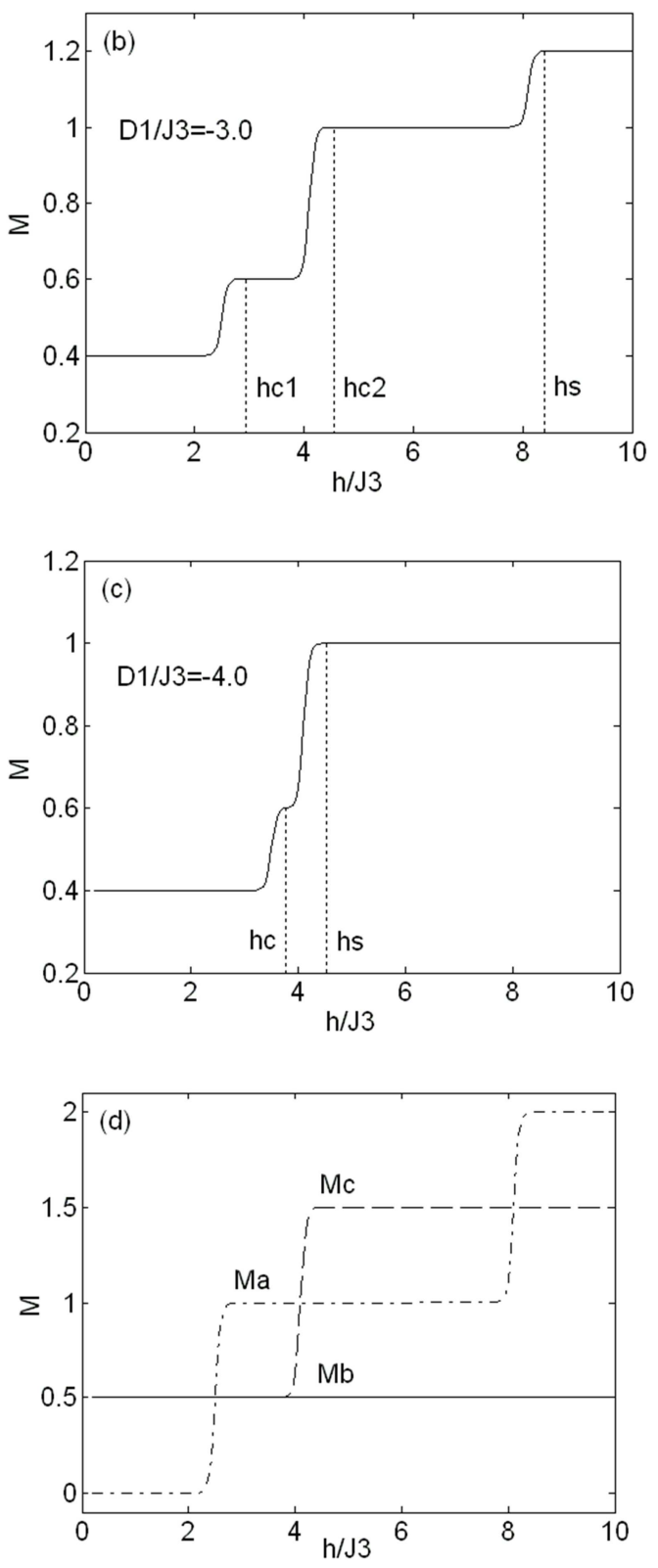

图3 改变D1/J3时的磁矩。

图3（a）中可以看到，当 $\mathrm{D}_{1} / \mathrm{J}_{3}$ 取 -0.5 时有三个磁化平 台, 即 $\mathrm{M}=0.6,0.8,1.2$, 对应的临界磁场 hc 为 1.45 , 饱 和磁场 hs 为 4.05 。图3 (b) 中可以看到, 当增加 $\left|D_{1 /} J_{3}\right|$ 到 $|-3.0|$ 时, 磁化曲线增加为四个磁化平台, 且平台对应的 磁矩也发生变化, 即 $\mathrm{M}=0.4,0.6,1,1.2$, 对应的临界磁 场 $\mathrm{hc}$ 为 $2.95,4.55$, 饱和磁场 $\mathrm{hs}$ 为 8.4 。图3 (c) 中可以 看到, 当增加 $\left|D_{1} / J_{3}\right|$ 到 $|-4.0|$ 时, 磁化曲线又减少为三个 磁化平台, 即 $\mathrm{M}=0.4,0.6,1$, 对应的临界磁场 hc 为 3.8 , 饱和磁场 $\mathrm{hs}$ 为 4.45 。为了进一步研究磁化平台产生时, 三 个子格对其的影响, 我们给出了 $\mathrm{D}_{1} / \mathrm{J}_{3}=-3.0$ 时三个子格的 磁矩, 见图3 (d), 点划线代表A子格的磁矩Ma, 实线代 表 $\mathrm{B}$ 子格的磁矩Mb，长虚线代表 $\mathrm{C}$ 子格的磁矩Mc。从图中可 以看到, $\mathrm{A}$ 子格有三个磁化平台, 即 $\mathrm{M}=0,1,2, \mathrm{C}$ 子格有 两个磁化平台, 即 $\mathrm{M}=0.5,1.5$, 子格 $\mathrm{B}$ 只有一条直线, 磁 化平台 $M b=0.5$ 。研究结果表明磁晶各向异性 $\mathrm{D}_{1} / \mathrm{J}_{3}$ 影响磁矩
曲线平台的个数和磁矩大小, 当 $\mathrm{D}_{1} / \mathrm{J}_{3}$ 取适当大时, 磁矩曲 线平台的个数最多; 对总的平均磁矩有主要贡献的是 $\mathrm{A}$ 子 格和 $\mathrm{C}$ 子格, 这是由于 $\mathrm{A}$ 子格和 $\mathrm{C}$ 子格的自旋 $\left(S_{i}^{z}=2, \sigma_{i 2}^{z}\right.$ $=3 / 2)$ 大于B子格的自旋 $\left(\sigma_{i 1}^{z}=1 / 2\right)$ 。

\section{2. 各物理参量对磁矩和磁化率的的影响}

图4（a）和（b）给出了交换作用 $\mathrm{J}_{1} / \mathrm{J}_{3}$ 取 $0.5 、 0.9$ 、 1. 3 和 1.8 时系统磁矩和磁化率随温度的变化曲线, 其它参 数选择为: $\mathrm{J}_{2} / \mathrm{J}_{3}=0.2, \mathrm{D}_{1} / \mathrm{J}_{3}=-2.1, \mathrm{D}_{2} / \mathrm{J}_{3}=-2.5, \mathrm{~h} / \mathrm{J}_{3}=2$ 。
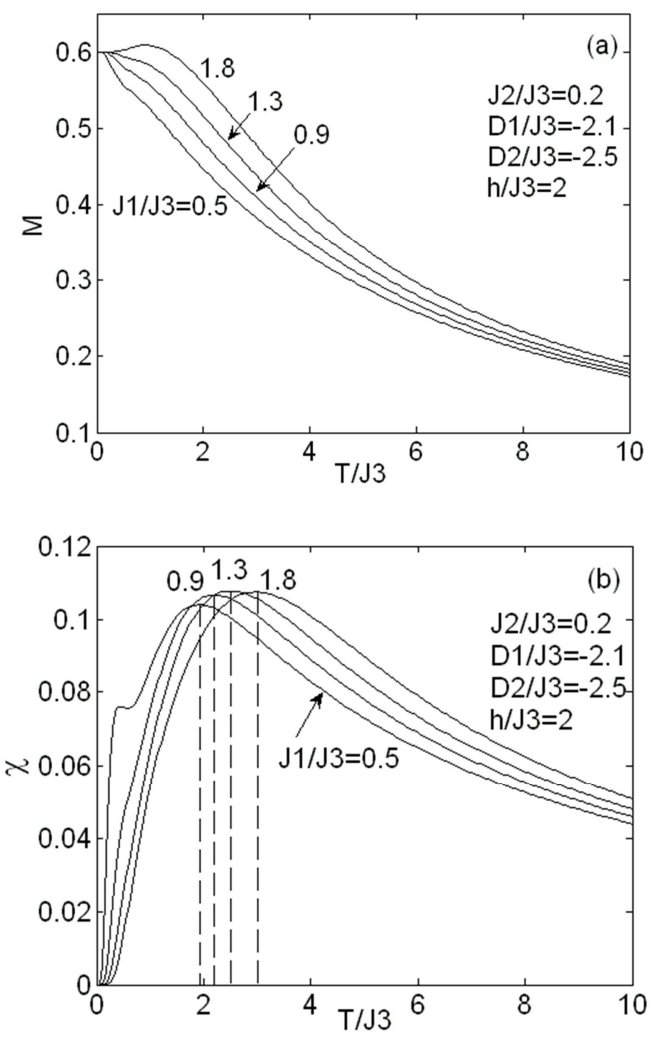

图4 改变 $\mathrm{J} 1 / \mathrm{J} 3$ 时的磁矩和磁化率。

图4（a）中可以看到，系统总的平均磁矩M随着温度 的增加而减小, 在同一温度下, 当 $\mathrm{J}_{1} / \mathrm{J}_{3}$ 从 0.5 增加到 1.8 时, 系统总的平均磁矩 $M$ 也随之增加, 在温度 $\mathrm{k}_{\mathrm{B}} \mathrm{T} / \mathrm{J}_{3}=0$ 时, 磁矩曲线的初始值均相同, 即 $\mathrm{M}=0.6$, 该初始值叫做饱和 磁化强度, 对应着图2（b）中的第二阶平台的磁化强度。 图4（b）中可以看到, 磁化率随温度变化的曲线会出现一 个峰值, 此时系统将无法发生磁化, 自旋磁有序结构将消 失, 系统由原来的铁磁性转变为超顺磁性。从图中可以看 到, 当交换作用 $\mathrm{J}_{1} / \mathrm{J}_{3}$ 从 0.5 增加到 1.8 时, 磁化率曲线的峰 值向右移动, 即向着温度升高的方向移动, 也就是说系统 由铁磁性转变为超顺磁性的温度升高。此外, 从图中可以 看到当温度 $\mathrm{k}_{\mathrm{B}} \mathrm{T} / \mathrm{J}_{3}=0$ 时, 系统的平均磁化率均为零。

图5 (a) 和 (b) 给出了 $\mathrm{A}$ 子格的磁晶各向异性 $\mathrm{D}_{1} / \mathrm{J}_{3}$ 取 $-0.2 、-0.6 、-1.8$ 和 -3.5 时系统磁矩和磁化率随温度的 变化曲线, 其它参数选择为: $\mathrm{J}_{1} / \mathrm{J}_{3}=0.3, \mathrm{~J}_{2} / \mathrm{J}_{3}=0.2$, $\mathrm{D}_{2} / \mathrm{J}_{3}=-2.5, \mathrm{~h} / \mathrm{J}_{3}=2$ 。 

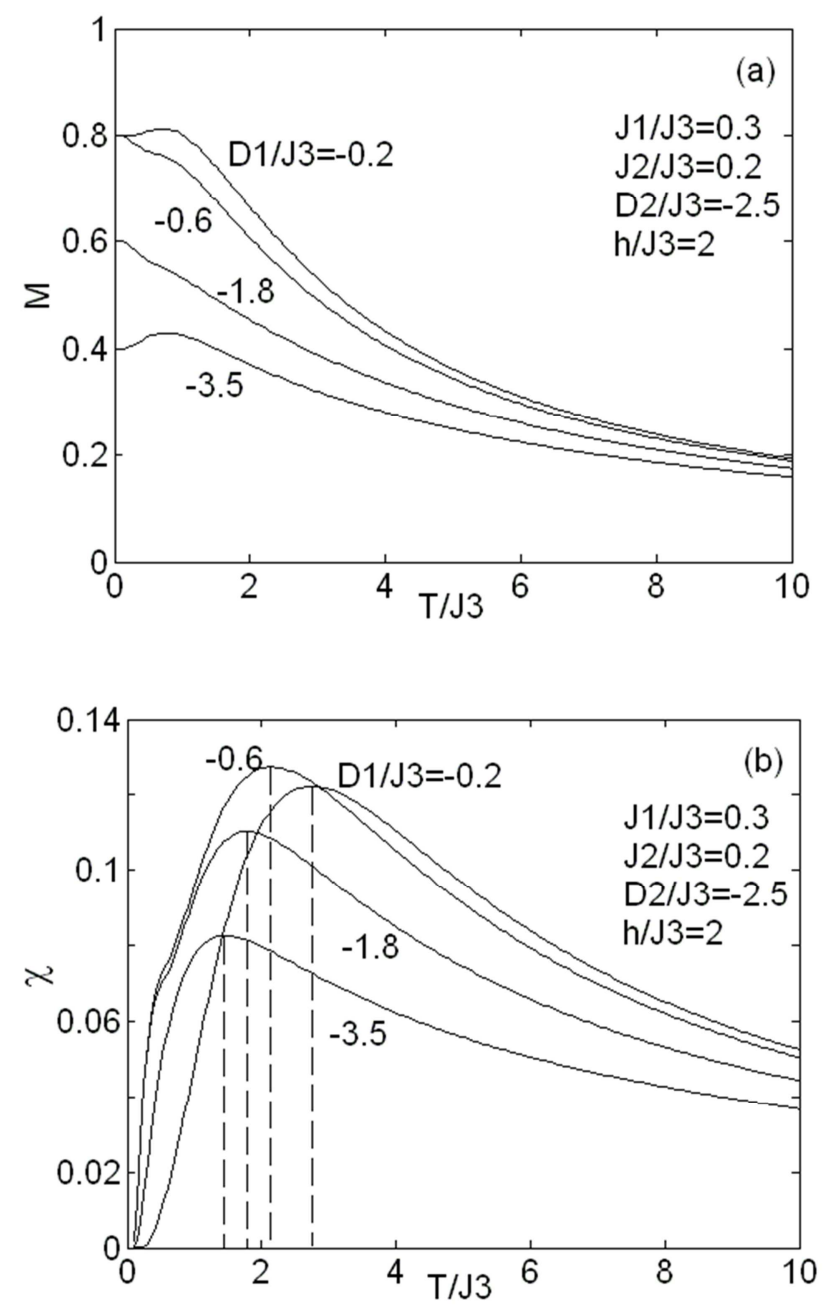

图5 改变D1/J3时的磁矩和磁化率。

从图5 (a) 中可以看到, 磁矩随温度变化的趋势与图 4 (a) 相同, 即随着温度的升高磁矩逐渐减小。在同一温 度下, 当 $A$ 子格晶场值 $\left|D_{1} / J_{3}\right|$ 从 $|-0.2|$ 增加到 $|-3.5|$ 时, 系统平均磁矩M逐渐变小, 且系统的饱和磁矩分布在固定 位置, 即 $\mathrm{M}=0.4,0.6$ 和 0.8 , 与磁化平台对应的磁化强度 相同。图5(b) 中可以看到, 当 $A$ 子格晶场值 $\left|D_{1} / J_{3}\right|$ 从 $|-0.2|$ 增加到 $|-3.5|$ 时, 磁化率曲线的峰值向温度降低的方向移 动, 且先增大后减小。也就是说, 随着A子格晶场值的增 加, 磁化率先增大后减小, 系统由铁磁性转变为超顺磁性 的温度逐渐降低。

图6 (a)-(d) 给出了交换作用 $\mathrm{J}_{2} / \mathrm{J}_{3}$ 取 $0.8 、 1.55 、 1.7$ 和1. 9 时系统磁矩和磁化率随温度的变化曲线, 其它参数 选择为: $\mathrm{J}_{1} / \mathrm{J}_{3}=0.3, \mathrm{D}_{1} / \mathrm{J}_{3}=-2.1, \mathrm{D}_{2} / \mathrm{J}_{3}=-2.5, \mathrm{~h} / \mathrm{J}_{3}=2$ 。

从图中可以看到, 图6 (a) 和 (b) 是系统磁矩随温 度的变化曲线, 图6 (c) 和 (d) 是磁化率随温度的变化 曲线。图6 (a) 中可以看到, 在同一温度下, 当交换作用 $\mathrm{J}_{2} / \mathrm{J}_{3}$ 从 0.8 增加到 1.7 时, 系统平均磁矩逐渐增大, 且饱和 磁化强度分布在 $M=0.6$ 和 1.2 , 对应着图 2 中的第二阶平台 和第四阶平台的磁化强度。图6 (b) 表示的是当 $\mathrm{J}_{2} / \mathrm{J}_{3}=1.55$ 时 $A 、 B 、 C$ 三个子格的磁矩Ma、Mb和Mc。
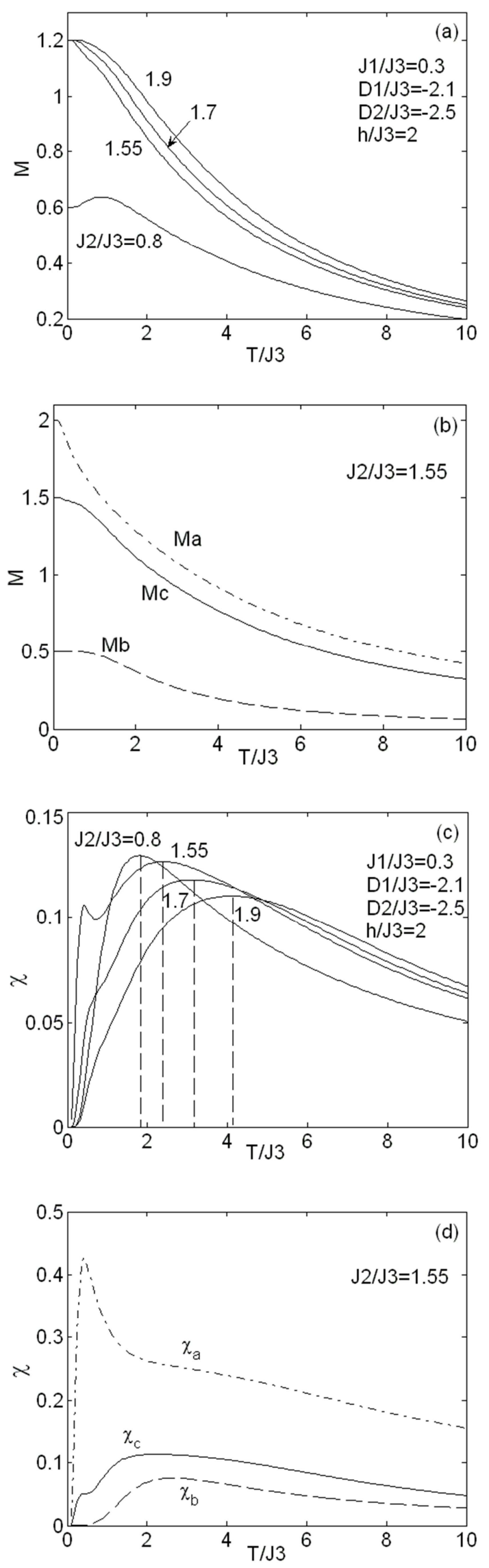

图6 改变 $\mathrm{J} 2 / \mathrm{J} 3$ 时的磁矩和磁化率。 
从图6（c）中可以看到, 当交换作用 $\mathrm{J}_{2} / \mathrm{J}_{3}$ 从 0.8 增加 到1. 7 时, 磁化率曲线的峰值向着温度升高的方向移动, 且逐渐减小, 即系统从铁磁性转变为超顺磁性的温度升高。 研究结果表明, 交换作用的增加会使系统由铁磁性转变为 超顺磁性的温度升高。图6 (d) 中可以看到 $\mathrm{B}$ 子格和 $\mathrm{C}$ 子格 的磁化率曲线峰值对应的温度相差不多, 而 $\mathrm{A}$ 子格的峰值 相比较B和C向左偏移很多, 合成之后总的磁化率如图7 (c) 中 $\mathrm{J}_{2} / \mathrm{J}_{3}=1.55$ 所示, 会发现在曲线峰值的前面出现一个小 的峰值, 这正是由于图6 (d) 中A子格磁化率峰值对应的 温度较低所致, 所以总的磁化率曲线可能会出现一个以上 的峰值。

图7给出了外磁场 $\mathrm{h} / \mathrm{J}_{3}$ 取 $1 、 2.5$ 和 3.5 时系统磁矩和磁 化率随温度的变化曲线, 其它参数选择为: $\mathrm{J}_{1} / \mathrm{J}_{3}=0.3$, $\mathrm{J}_{2} / \mathrm{J}_{3}=0.2, \mathrm{D}_{1} / \mathrm{J}_{3}=-2.1, \mathrm{D}_{2} / \mathrm{J}_{3}=-2.5$ 。

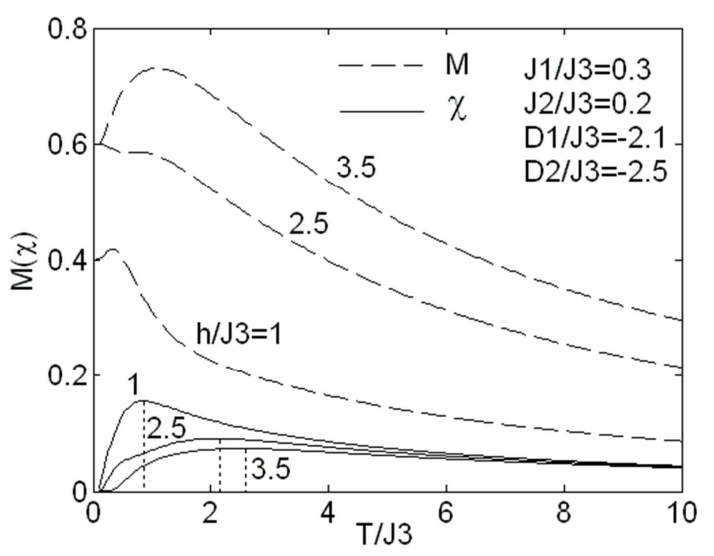

图7 改变h/J3时的磁矩和磁化率。

从图中可以看到, 在同一温度下, 随着外磁场 $\mathrm{h} / \mathrm{J}_{3}$ 的 增加, 系统平均磁矩逐渐增大, 磁化率曲线的峰值向温度 升高的方向移动。在温度 $\mathrm{k}_{\mathrm{B}} \mathrm{T} / \mathrm{J}_{3}=0$ 时, 饱和磁矩有固定值, 即外磁场 $\mathrm{h} / \mathrm{J}_{3}=1$ 时, 饱和磁矩为 $\mathrm{M}=0.4$, 当外磁场 $\mathrm{h} / \mathrm{J}_{3}=2.5$ 和3. 5 时, 饱和磁矩为 $M=0.6$, 对应着图 2 中的第一、二阶 平台的磁化强度。结果表明在不同的外磁场下磁化率的初 始值均为零, 结论与本节中图4-图6相一致。

\section{4. 结论}

本文利用相关有效场理论研究低温情况下的混自旋 2 , $1 / 2,3 / 2$ Ising钻石链的磁化平台、磁矩和磁化率。数值 计算研究结果表明, 随着交换作用的增大, 磁化曲线平台 阶梯数减少, 第一个临界磁场hc和饱和磁场hs减小, 第二 个临界磁场保持不变; 随着晶场值的增大, 磁化曲线平台 阶梯数先增多后减少。系统磁矩随温度的增加而减小, 在 同一温度下, 磁矩随交换作用的增大而增大, 随晶场值的 增大而减小, 随外磁场的增大而增大。磁矩有着固定的饱 和磁化强度, 对应着磁化平台的磁化强度。磁化率峰值随 交换作用增大向温度升高的方向移动，随晶场值的增大向 温度降低的方向移动, 随外磁场h的增大向温度升高的方
向移动。探索在晶场作用下有机和无机材料中的铁磁钻石 链结构模型在磁学研究中将会开启一个新的领域。

\section{参考文献}

[1] C. W. Rischau, B. Leridon, D. Colson, A. Forget, P. Monod, $\mathrm{BaCu}_{3} \mathrm{O}_{4}$ : High-temperature magnetic order in one-dimensional S=1/2 diamond chains [J]. Phys. Rev. B. 2012, 85: 134518 .

[2] 冯丽雅, 辛子华, 王吴蹈. 一维钻石链反铁磁Ising模型磁 化的模拟 [J]. 计算物理, 2010, 27 (4) :613。

[3] Z. H. Xin, S. Chen, C. Y. Zhang, J. Spin frustration-related behavior of mixed spin diamond chain[J]. J. Magn. Magn. Mater, 2012, 324: 3704.

[4] K. Okamoto1, T. Tonegawa, M. Kaburagi. Magnetic properties of the $\mathrm{s}=1 / 2$ distroted diamond chain at $\mathrm{T}=0$ [J]. J. Phys. : Condens. Matter, 2003, 15(35) : 5979.

[5] M. Drillon, E. Coronado, M. Belaiche, et al. Low-dimensional magnetic systems; from 1D to 3D ferrimagnets [J]. J. Appl. Phys. 1988, 63(8) : 3551.

[6] A. Honecker, S. J. Hu, R. Peters, et al. Dynamic and thermodynamic properties of the generalized diamond chain model for azurite [J]. J. Phys. : Condens. Matter, 2011, $23(16)$ : 164211.

[7] J. J. Jiang, Y. J. Liu, F. Tang, et al. The exact ground state and the origin of the apin gap in the distroted mixed $\operatorname{spin}(1,1 / 2)$ diamond chain $[\mathrm{J}]$. Physica B, 2012, $407(24): 4810$.

[8] T. M. Cheng, Y. M. Ma, L. Li, et al. Magnetism and thermodynamic properties of a spin $-1 / 2$ ferrimagnetic diamond $X Y$ chain in magnetic fields at finite temperatures $[\mathrm{J}]$. Journal of the Korean Physical Society, 2013, 62(1): 104-105.

[9] B. Lisnyi, J. Strecka. Ground-state phase diagram and magnetization process of the exactly solved mixed spin - $(1,1 / 2)$ Ising diamondn chain [J]. J. Magn. Magn. Mater, 2013, 346: 78-83.

[10] N. Ananikian, H. Lazaryan, M. Nalbandyan. Magnetic and quantum entanglement properties of the distorted diamond chain model for azurite $[\mathrm{J}]$. Eur. Phys. J. B, 2012, $85(7): 223$.

[11] W. Jiang, L. M. Liu, X. X. Li, et al. Magnetization plateaus of a ferrimagnetic nanoparticle in the presence of single-ion anisotropies [J]. Physica B, 2012, $407(19): 3933$. 